\title{
A 46-year-old female with dyspnoea, stridor and chronic cough
}

\author{
N. Maimon*, T. Marras*, D. Hwang*, N. Paul ${ }^{*}$, S. Keshavjee ${ }^{+}$and C.K.N. Chan*
}

\section{CASE HISTORY}

A 46-yr-old female presented to the chest clinic with chronic dry cough and increasing dyspnoea on exertion. The patient's symptoms had deteriorated over the past 15 months and, at presentation, the patient developed dyspnoea after only 50 yards of walking. She had experienced multiple emergency department visits and frequent admissions to the hospital because of breathing difficulties. Also, during the last year, she experienced recurrent respiratory infections with a frequency of one to two infections per month. The patient received a diagnosis of bronchial asthma and was treated with short courses of systemic steroids, multiple inhalers and courses of antibiotics; resulting in only mild and temporary improvement in her symptoms. Pulmonary function tests (PFTs) 15 months earlier had shown a forced expiratory volume in one second (FEV1) of $1.5 \mathrm{~L}$ (84\% predicted).

The patient's past medical history was unremarkable. Specifically, there was no history of childhood asthma or frequent infections. She was an ex-smoker with 15 pack-yrs. Family history, environmental allergies and occupational exposures were all unremarkable. At presentation her medica-

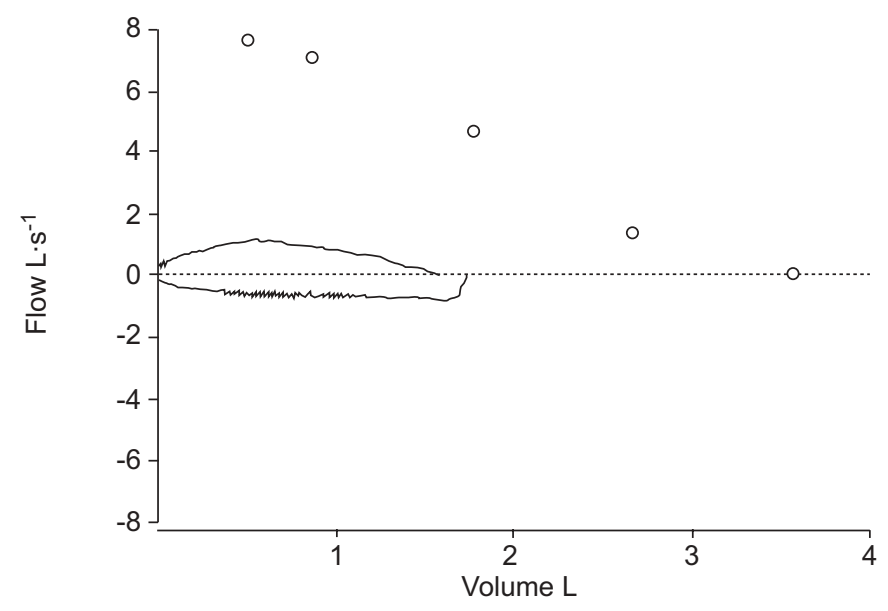

FIGURE 1. A flow-volume loop. $\bigcirc$ : normal predicted values for the patient.
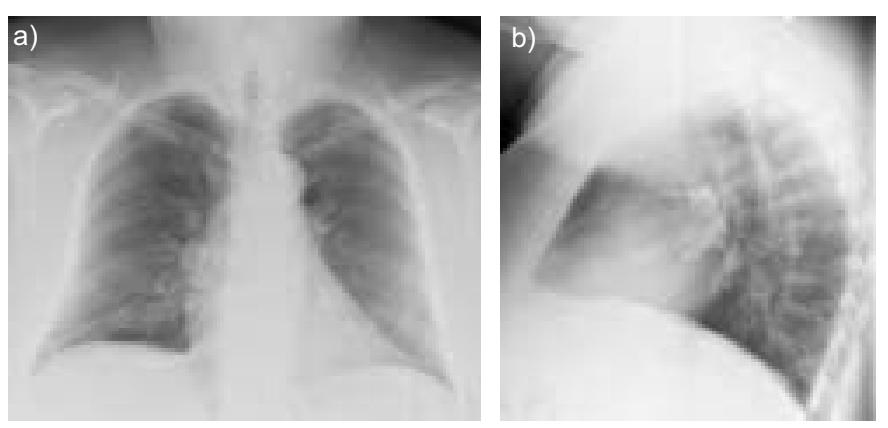

FIGURE 2. Chest radiograph obtained at presentation of the a) posterioranterior and b) lateral view.

tions included salmeterol-fluticasone inhaler, tiotropium, montelukast, prednisone $30 \mathrm{mg}$ daily and pantoprazole.

The patient appeared well with a blood pressure of $130 / 80$ $\mathrm{mmHg}$, heart rate of $80 \cdot \mathrm{min}^{-1}$ and regular, respiratory rate of $18 \cdot \mathrm{min}^{-1}$. Her saturation on room air was at $95 \%$. Head and neck examination did not demonstrate lymphadenopathy or signs of chondral inflammation. Her cardiovascular examination revealed a normal jugular vein pulse with normal heart sounds and no pedal oedema. Respiratory examination demonstrated absence of clubbing and no evidence of wheezing or crackle. However, forced expiration produced an audible stridor sound.

Spirometry demonstrated a forced vital capacity (FVC) of $1.7 \mathrm{~L}$ (75\% pred), FEV1 0.65 L (25\% pred), and FEV1/FVC 35\%. A flow/volume curve is shown in figure 1 . Diffusing capacity of the lung for carbon monoxide was 16.5 (95\% pred). On presentation, chest radiography and computed tomography (CT) were performed (figs 2 and 3, respectively) followed by helical thoracic CT (fig. 4).

The patient underwent bronchoscopy, which showed abnormal tracheal anatomy with a severely thickened, inflamed, narrowed and collapsible trachea. Bronchial washings showed negative results by cytology and microbiology. A transtracheal biopsy was also performed (fig. 5).

Divisions of *Respirology, " Pathology, "Radiology, and +Thoracic Surgery, Dept of Medicine, Toronto General Hospital, University Health Network, University of Toronto, Toronto, Canada.

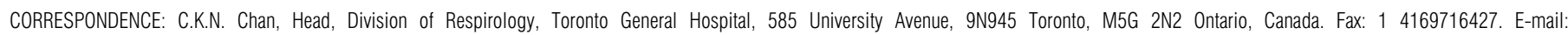
charles.chan@uhn.on.ca 

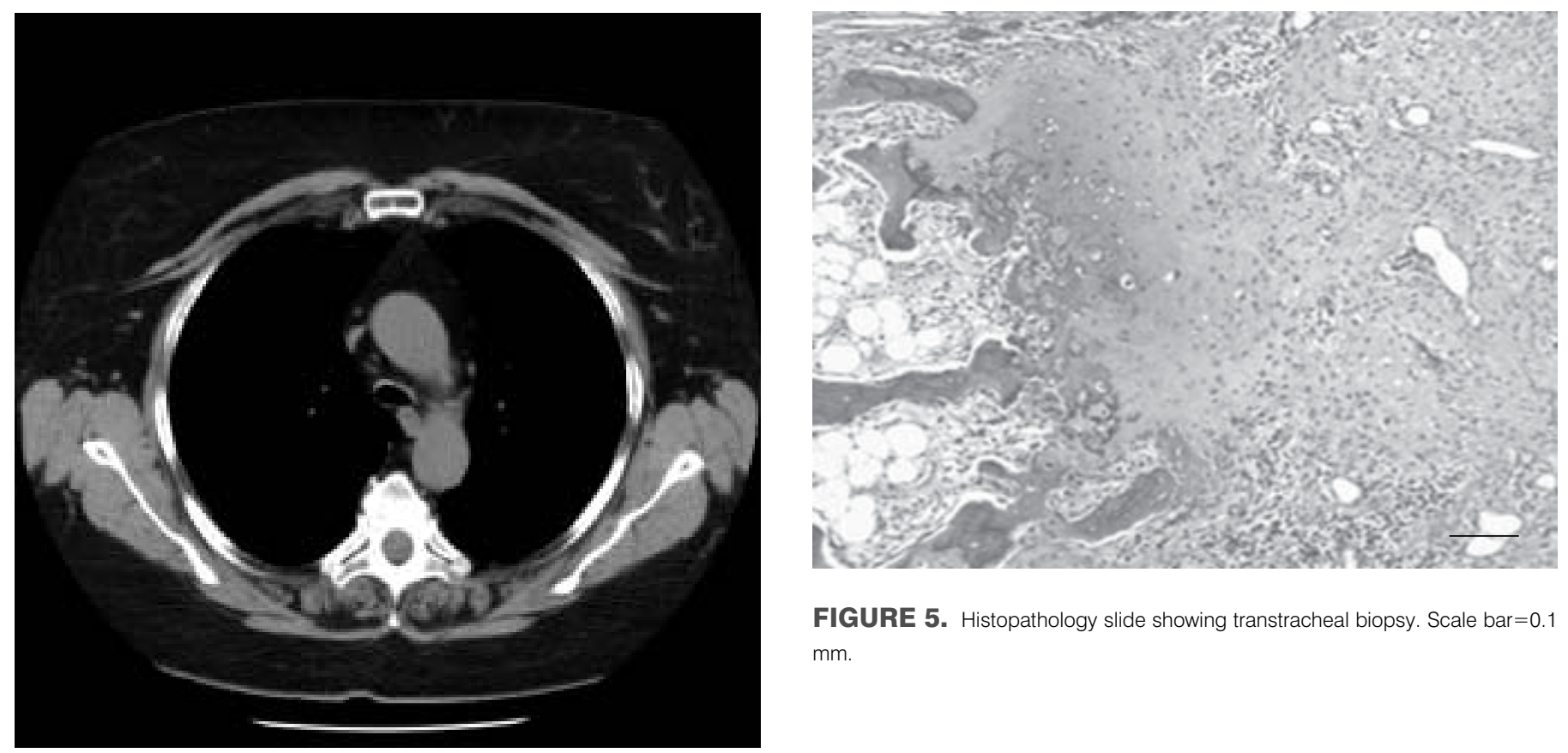

FIGURE 5. Histopathology slide showing transtracheal biopsy. Scale bar $=0.1$ $\mathrm{mm}$.

FIGURE 3. Computed tomography obtained on presentation of the chest at the carina level.
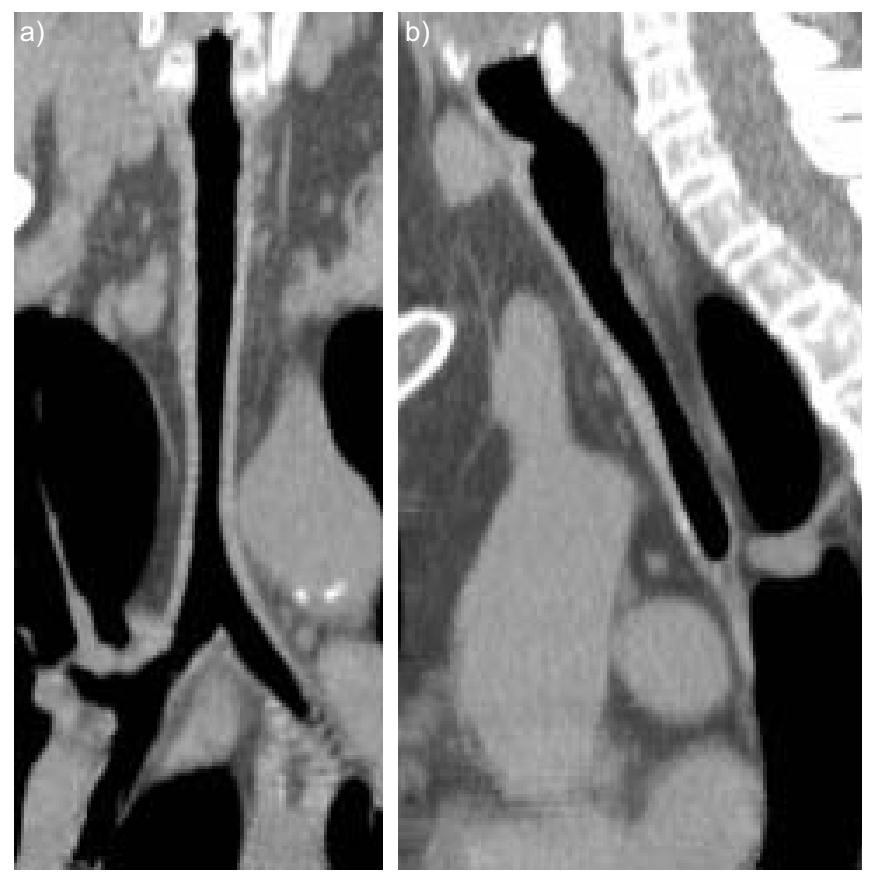

FIGURE 4. Helical computed tomography obtained at presentation of the a) coronal and b) sagittal view.

BEFORE TURNING THE PAGE INTERPRET THE FLOW-VOLUME LOOP, CHEST RADIOGRAPHS, THE COMPUTED TOMOGRAPHY SCANS AND THE HISTOLOGY SLIDE, AND SUGGEST A DIAGNOSIS. 


\section{INTERPRETATION}

The flow-volume loop (fig. 1) shows fixed air flow limitation on both inspiration and expiration. The chest radiograph, both posterior-anterior and lateral views, shows there is narrowing of the mid and distal trachea (fig. 2).

The CT scan of the thorax at the carina level, in figure 3, demonstrates thickening of the anterior cartilaginous tracheal wall $(3 \mathrm{~mm})$ and narrowing of the luminal diameter $(7-8 \mathrm{~mm})$. No mediastinal or hilar lymphadenopathy was noted and the lungs fields are unremarkable.

The helical CT scan of the coronal and sagittal view (fig. 4) demonstrates diffuse narrowing of the tracheal lumen and thickening of the tracheal wall.

The histopathological slide of the transtracheal biopsy (fig. 5) indicates the presence of chronic inflammatory infiltrates with cartilage destruction and fibrosis, which are associated with reactive changes and osseous metaplasia.

\section{Diagnosis: Respiratory relapsing polychondritis.}

\section{Treatment and clinical course}

Transtracheal biopsy showed evidence of cartilage destruction and fibrosis, which is consistent with the diagnosis of relapsing polychondritis. The diagnosis of relapsing polychondritis was made according to the modified diagnostic criteria based on tracheal involvement and positive histology.

The patient was started on prednisone $60 \mathrm{mg}$ daily, as well as calcium supplements. Upon follow-up 4 weeks later, she had noticed increased exercise capacity. A chest CT scan demonstrated minimal decrease in her tracheal narrowing, and there was minimal improvement in her PFTs. She was put on a very slow steroid taper ( $5 \mathrm{mg}$ every 6 weeks) with the additional maintenance of cyclophosphamide at a dose of $100 \mathrm{mg} \cdot$ day $^{-1}$. She was followed at 4-week intervals and continued to have exercise dyspnoea and stridor. A repeat bronchoscopy 8 weeks later showed persistent inflamed narrowed and collapsible trachea. FEV1 deteriorated further and the patient underwent stent insertion in the trachea and main bronchi. Although the procedure was successful, it was difficult to extubate the patient and while in the intensive care unit she acquired severe pneumonia and died 2 weeks later. An autopsy confirmed the diagnosis of respiratory chondritis and the cause of death was severe pneumonia.

\section{DISCUSSION}

Relapsing polychondritis is an uncommon multisystem disease, which can be life threatening and debilitating. It is characterised by recurrent episodes of inflammation and destruction of all types of cartilaginous tissue [1]. The organs typically affected are the external ear, nose, larynx, trachea and major bronchi. There is equal distribution between sexes; however, females suffer more from the respiratory complications of the disease in a ratio of 2:1 [1-3]. The median age for diagnosis is 47 yrs and it is empirically established by the presence of three or more of any of the following features: 1) bilateral auricular chondritis; 2) nonerosive seronegative inflammatory arthritis; 3) nasal chondritis; 4) ocular inflammation; 5) respiratory tract chondritis; or 6) audio-vestibular damage $[1,2]$.
Involvement of the cartilaginous structures of the respiratory tract, although uncommon at presentation, occurs in almost half of relapsing polychondritis patients during the course of their illness [1]. Respiratory involvement is a poor prognostic sign and accounts for $>50 \%$ of relapsing polychondritis related deaths. Progression of tracheal disease leads to fibrotic changes and diffuse narrowing of the main airway and severe airflow impairment. Persistent bronchial cartilage inflammation may cause impairment of mucociliary function. Both factors, in addition to the use of steroids and other immunosuppressive drugs, predispose to recurrent respiratory tract infections. Pulmonary parenchymal involvement is not characteristic of relapsing polychondritis $[1,3,4]$.

Most patients with respiratory involvement assume a fluctuating but progressive course. The polycyclic bouts of inflammation eventually lead to permanent destruction of the large airways. In severe cases, persistent inflammation can cause acute airway narrowing and destroy the cartilaginous rings, which creates luminal collapse. Obstruction may also be induced iatrogenically by bronchoscopy or tracheostomy. Intubation may be difficult and dangerous because of a small glottis caused by oedema or cartilage destruction. Another uncommon manifestation of respiratory polychondritis is the involvement of costosternal cartilages, which may lead to costochondritis that may further impair breathing $[3,4]$.

Conventional radiographs and CT scans identify laryngotracheal lesions, and thin-section CT defines abnormalities in both the trachea and lobar bronchi. Three-dimensional or spiral magnetic resonance imaging may provide better resolution $[5,6]$. The nature of airway obstruction, whether fixed or dynamic, and the location, whether intra- or extrathoracic, can be assessed by PFTs, especially flow-volume loops. PFTs are useful tools for monitoring change over time [7]. In addition, CT scans and PFTs may detect otherwise asymptomatic airway involvement. Bronchoscopy may also be informative but carries a risk for exacerbating airway inflammation. The airways mucosa is typically inflamed and the involved segments are narrowed but there is nothing pathognomonic on direct visualisation.

When the respiratory complications appear in a patient with established relapsing polychondritis, the diagnosis is obvious. However, when the initial manifestation of relapsing polychondritis is respiratory there will be a delay in diagnosis in most cases. The clinical symptoms include cough and dyspnoea and have partial response to steroids, are nonspecific and usually attributed to more common diseases, such as asthma or chronic obstructive pulmonary disease. A chest radiograph, that in most cases will be normal, can also be misleading. The course is often progressive over several months. In some patients, constitutional symptoms, such as weight loss, fever and fatigue, may suggest a more systemic condition. Physical examination is often unremarkable, and when there is an abnormal finding it is most commonly stridor. As the disease progresses, there is further deterioration in FEV1, which is associated with the typical impairment of the inspiratory and expiratory curves. In such cases of suspected upper airway obstruction, a CT scan of the chest can demonstrate tracheal narrowing. In this instance the next diagnostic test would be bronchoscopy [3]. 
The differential diagnosis of tracheal wall narrowing includes infectious, inflammatory and neoplastic processes. In the paediatric population, infectious processes, such as acute tracheobronchitis (croup) or acute bacterial membranous tracheitis, which is less common, may cause stridor and tracheal narrowing. In the adult population, tuberculous tracheitis is relatively rare and is almost always associated with pulmonary cavitations. However, in the healing stage of the disease it can cause tracheal fibrosis with smooth narrowing of the wall. The most common form of primary pulmonary amyloidosis is tracheobronchial, and patients may present with multiple or localised masses on the tracheal wall or diffuse narrowing of the tracheal wall. The difference in the radiological appearance is the circumference of the amyloid lesions compared with the tendency to spare the posterior membranous portion in relapsing polychondritis disease. Sarcoidosis may also be reported to cause isolated tracheal narrowing.

The histopathological findings of tracheal biopsy in relapsing polychondritis typically include loss of basophilic staining of the cartilage matrix, perichondrial round cell infiltration and destruction of cartilage with fibrous replacement. Endobronchial biopsy tends not to provide cartilaginous materials for a firm diagnosis. Partial tracheal resection may yield sufficient materials for pathological confirmation [3].

Treatment with corticosteroids, alone or in combination with other immunosuppressive therapy, has been traditionally employed. Although its efficacy has not been assessed in randomised trials, it is the most effective treatment and can even lead to a dramatic response. Optimal dose and duration of therapy has again not been systematically evaluated. A reasonable regimen is $1 \mathrm{mg} \cdot \mathrm{kg}^{-1}$ once daily for $8-12$ weeks and re-evaluation of the patient with tapering to the lowest dose possible while maintaining disease control. Lifetime treatment may be necessary in patients with aggressive disease. Traditionally, drugs such as cyclophosphamide, dapsone and azathioprine have provided additional therapy with steroid sparing affect. Recent experience in an uncontrolled trial suggests that methotrexate and anti-tumour necrosis factor- $\alpha$ may be helpful [2].

The possible outcomes of respiratory involvement of relapsing polychondritis include spontaneous remission, stabilisation following treatment with corticosteroids or other immunosuppressive therapy, progression to life-threatening tracheal obstruction, or progression to tracheal obstruction and death despite interventional bronchoscopies with stents insertion [8]. Secondary infections are another potential complication of the disease, and these were unfortunately the cause of death in the current authors' patient.

\section{REFERENCES}

1 McAdam LP, O'Hanlan MA, Bluestone R, Pearson CM. Relapsing polychondritis: prospective study of 23 patients and a review of the literature. Medicine 1976; 55: 193-215.

2 Trentham DE, Le CH. Relapsing polychondritis. Ann Intern Med 1998; 129: 114-122.

3 Tillie-Leblond I, Wallaert B, Leblond D, et al. Respiratory involvement in relapsing polychondritis. Clinical, functional, endoscopic, and radiographic evaluations. Medicine 1998; 77: 168-176.

4 Faul JL, Kee ST, Rizk NW. Endobronchial stenting for severe airway obstruction in relapsing polychondritis. Chest 1999; 116: 825-827.

5 Mohsenifar Z, Tashkin DP, Carson SA, Bellamy PE. Pulmonary function in patients with relapsing polychondritis. Chest 1982; 81: 711-717.

6 Eng J, Sabanathan S. Airway complications in relapsing polychondritis. Ann Thoracic Surg 1991; 51: 686-692.

7 Booth A, Dieppe PA, Goddard PL, Watt I. The radiological manifestations of relapsing polychondritis. Clin Radiol 1989; 40: 147-149.

8 Port JL, Khan A, Barbu RR. Computed tomography of relapsing polychondritis. Comput Med Imaging Graph 1993; 17: 119-123. 